\title{
Beta function quintessence cosmological parameters and fundamental constants - II. Exponential and logarithmic dark energy potentials
}

\author{
Rodger I. Thompson ${ }^{\star}$ \\ Steward Observatory, University of Arizona, Tucson, AZ 85721, USA
}

Accepted 2018 October 29. Received 2018 October 26; in original form 2018 September 27

\begin{abstract}
This paper uses the beta function formalism to extend the analysis of quintessence cosmological parameters to the logarithmic and exponential dark energy potentials. The previous paper demonstrated the formalism using power and inverse power potentials. The essentially identical evolution of the Hubble parameter for all of the quintessence cases and $\Lambda \mathrm{CDM}$ is attributed to the flatness of the quintessence dark energy potentials in the dark energy dominated era. The Hubble parameter is therefore incapable of discriminating between static and dynamic dark energy. Unlike the other three potentials considered in the two papers the logarithmic dark energy potential requires a numerical integration in the formula for the superpotential rather than being an analytic function. The dark energy equation of state and the fundamental constants continue to be good discriminators between static and dynamical dark energy. A new analysis of quintessence with all four of the potentials relative the swampland conjectures indicates that the conjecture on the change in the scalar field is satisfied but that the conjecture on the change of the potential is not.
\end{abstract}

Key words: cosmological parameters - dark energy - early Universe.

\section{INTRODUCTION}

This is the second of two papers using the beta function methodology to produce accurate analytic solutions from model dark energy potentials in a quintessence cosmology. The first paper (Thompson 2018), hereinafter paper I, examined solutions for power and inverse power-law potentials. This work extends the analysis to logarithmic and exponential potentials. The analytic nature of the solutions provides the means to calculate solutions for other values of the input parameters such as $H_{0}$ and $\Omega_{m_{0}}$ in a flat universe for comparison with observations.

Exact analytic solutions for specific dark energy potentials are often mathematically intractable (Narain 2017) but the beta function formalism (Binetruy et al. 2015; Cicciarella \& Pieroni 2017) provides a method for achieving accurate analytic solutions using beta potentials $V_{\mathrm{b}}(\phi)$ that are accurate, but not exact, representations of model potentials $V_{\mathrm{m}}(\phi)$. In many cases numerical calculations can provide solutions for specific cases. Such solutions, however, often neither readily reveal the basic physics in play nor do they provide easily calculable solutions for alternative input parameters. The particular potentials examined here are the logarithmic

$V_{\mathrm{m}}(\phi) \propto\left(\frac{\ln (\phi)}{\ln \left(\phi_{0}\right)}\right)^{\beta_{l}}$

\footnotetext{
^E-mail: rit@email.arizona.edu
}

and exponential

$$
V_{\mathrm{m}}(\phi) \propto \exp \left[-\beta_{e}\left(\phi-\phi_{0}\right)\right]
$$

potentials, where $\beta_{l}$ and $\beta_{e}$ are real, positive constants.

The methodology follows the descriptions in Binetruy et al. (2015) and Cicciarella \& Pieroni (2017), particularly Cicciarella \& Pieroni (2017) who explicitly include matter as well as dark energy. The details of the analysis are given in paper I and will not be repeated here except for clarity. This work concentrates on the 'late time' evolution of the Universe which is taken to be the time between a scale factor of 0.1 and 1.0 corresponding to redshifts between zero and nine. A flat Universe is assumed with $H_{0}=70 \mathrm{~km} \mathrm{~s}^{-1}$ per megaparsec. The current ratio of the dark energy density to the critical density $\Omega_{\phi_{0}}$ is set to 0.7 where $\phi_{0}$ is the current value of the scalar $\phi$. The current values of the dark energy equation of state are set to $w_{0}=(-0.98,-0.96,-0.94,-0.92,-0.90)$ as was done in paper I. The last two values of $w_{0}$ are unlikely but are included to determine the limits on the validity of the solutions. In the exponential model potential the value of $w_{0}$ determines the value of $\beta_{e}$ removing one degree of freedom. In paper I $\kappa=\frac{\sqrt{8 \pi}}{m_{p l}}$ was set to one, however, in this paper natural units are used with $m_{\mathrm{pl}}$, the Planck mass, set to one. This makes the units of the scalar $\phi$ the Planck mass rather than $1 / \kappa$. A section on where the quintessence cases considered here and in paper I dwell relative to the swampland conjectures has also been added. 


\section{QUINTESSENCE}

Quintessence is characterized by an action of the form

$S=\int \mathrm{d}^{4} x \sqrt{-g}\left[\frac{R}{2}-\frac{1}{2} g^{\mu \nu} \partial_{\mu} \partial_{\nu} \phi-V(\phi)\right]+S_{\mathrm{m}}$,

where $R$ is the Ricci scalar, $g$ is the determinant of the metric $g^{\mu \nu}$, $V(\phi)$ is the dark energy potential, and, $S_{\mathrm{m}}$ is the action of the matter fluid. Different types of quintessence are defined by different forms of the dark energy potential. The quintessence dark energy density, $\rho_{\phi}$, and pressure, $p_{\phi}$, are given by

$\rho_{\phi} \equiv \frac{\dot{\phi}^{2}}{2}+V(\phi), \quad p_{\phi} \equiv \frac{\dot{\phi}^{2}}{2}-V(\phi)$.

\section{THE BETA FUNCTION}

The beta function is defined as the derivative of the scalar $\phi$ with respect to the natural $\log$ of the scale factor $a$ (Binetruy et al. 2015)

$\beta(\phi) \equiv \frac{\kappa \mathrm{d} \phi}{\mathrm{d} \ln (a)}=\kappa \phi^{\prime}$,

where $\kappa=\frac{\sqrt{8 \pi}}{m_{p l}}$ and the prime on the right hand term denotes the derivative with respect to the natural $\log$ of the scale factor except when it denotes the integration variable inside an integral as in equation (11). As noted in the introduction paper I set $\kappa$ to one as is often done in the cosmological literature. Here instead the Planck mass is set to one leading to the scalar $\phi$ being expressed in units of the Planck mass, a difference of $\sqrt{8 \pi} \approx 5$ from paper I. In the following $k$ is used to denote $\sqrt{8 \pi}$ in an equation. Note that $\phi$ now has the dimensions of $m_{\mathrm{pl}}$ and that $\kappa \phi$ is dimensionless.

The dark energy equation of state $w=\frac{p_{\phi}}{\rho_{\phi}}$ for quintessence is given by Nunes \& Lidsey (2004)

$w+1=\frac{k^{2} \phi^{\prime 2}}{3 \Omega_{\phi}}=\frac{k^{2} \beta^{2}(\phi)}{3 \Omega_{\phi}}$

For the logarithmic potentials this equation provides the boundary condition to determine the current value of the scalar $\phi_{0}$. For the exponential potential equation (6) determines $\beta_{\mathrm{e}}$ as discussed in Section 4.

The beta function is not an arbitrary function of $\phi$ and $a$, but is determined the model dark energy potential $V_{\mathrm{m}}(\phi)$ such that (Cicciarella \& Pieroni 2017)

$V_{\mathrm{m}}(\phi)=\exp \left\{-\int k \beta(\phi) \mathrm{d} k \phi\right\}$.

\subsection{Beta functions from the potentials}

From equation (7) the appropriate beta function is the logarithmic derivative of the potential. Using the potentials listed in the introduction the logarithmic beta function is

$\beta(\phi)=\left(\frac{-\beta_{l}}{k \phi \ln (k \phi)}\right)$.

The exponential beta function is simply

$\beta(\phi)=\frac{\beta_{\mathrm{e}}}{k}$.

Five $\beta_{l}$ values are considered, the integers one through five. The $\beta_{\mathrm{e}}$ values are set by the five values of $w_{0}$.

\section{EVOLUTION OF THE SCALAR}

An important feature of the beta function formalism is that the specification of the beta function, along with a boundary condition determines the evolution of the scalar with respect to the scale factor $\phi(a)$.

\subsection{The scalar as a function of the scale factor (logarithmic)}

The beta function, equation (5), provides the differential equation for $\phi$ as a function of the scalar $a$. For the logarithmic potential

$k^{2} \phi \ln (k \phi) \mathrm{d} \phi=-\beta_{l} \mathrm{~d} \ln (a)$.

Integrating both sides

$\int_{\phi_{0}}^{\phi} k^{2} \phi^{\prime} \ln \left(k \phi^{\prime}\right) \mathrm{d} \phi^{\prime}=-\beta_{l} \int_{1}^{a} \mathrm{~d} \ln \left(a^{\prime}\right)$

gives

$\frac{k^{2} \phi^{2}}{2}\left(\ln (k \phi)-\frac{1}{2}\right)=-\beta_{l} \ln (a)+\frac{k^{2} \phi_{0}^{2}}{2}\left(\ln \left(k \phi_{0}\right)-\frac{1}{2}\right)$,

where $\phi_{0}$ is the current value of the scalar. Denoting the right hand term of the equation by $Q$ the scalar is given by

$k \phi= \pm \sqrt{\frac{2 Q}{\operatorname{PL}\left(\frac{2 Q}{\mathrm{e}}\right)}}$.

The term PL in equation (13) stands for the Product Log, more commonly known as the Lambert $W(x)$ function, the solution to $W e^{W}=x$. Here the Product Log term, used by Mathematica, is retained to avoid confusion with the superpotential $W(\phi)$ introduced later. The value of $\phi_{0}$ is determined by the current value of the dark energy equation of state $w_{0}$ using equation (6)

$k \phi_{0} \ln \left(k \phi_{0}\right)=\frac{ \pm \beta_{l}}{\sqrt{3 \Omega_{\phi_{0}}\left(w_{0}+1\right)}}$,

where $\Omega_{\phi_{0}}$ is the current ratio of the dark energy density to the critical density. The solution to equation (14) again uses the PL function

$k \phi_{0}=\frac{\frac{ \pm \beta_{l}}{\sqrt{3 \Omega_{\phi_{0}}\left(w_{0}+1\right)}}}{\operatorname{PL}\left(\frac{ \pm \beta_{l}}{\sqrt{3 \Omega_{\phi_{0}}\left(w_{0}+1\right)}}\right)}$.

The Product Log does not have positive real solutions for negative arguments. The definition of the logarithmic beta function assumes that $\beta_{l}$ is a positive real number, therefore, the positive square root is chosen in equations (13)-(15). None of the three equations accommodate phantom solutions where $(w+1)<0$.

Figs 1 and 2 show the evolution of the scalar $\phi$ for the logarithmic beta function with $\beta_{l}$ held constant at 3 in Fig. 1 for the five values of $w_{0}$ and $w_{0}$ is held constant at -0.94 in Fig. 2 for the five values of $\beta_{l}$. Even though $\phi_{0}$ changes significantly with the value of $\beta_{l}$, the scalar $\phi$ evolves relatively little over $a$ between 0.1 and 1 .

\subsection{The scalar as a function of the scale factor (exponential)}

The exponential potential, $V(\phi) \propto \exp \left[\beta_{\mathrm{e}}\left(\phi-\phi_{0}\right)\right]$ is the dark energy potential for slow roll quintessence when the first slow roll parameter, $\frac{1}{V} \frac{\mathrm{d} V}{\mathrm{~d} \phi}$ is held constant eg. Scherrer \& Sen (2008). The beta function for the exponential potential, $\beta_{\mathrm{e}}$, is unique in that it is a constant and not a function of $\phi$. Unlike all of the previous cases $\beta_{\mathrm{e}}$ cannot be set arbitrarily. The value of $\beta_{\mathrm{e}}$ is set by equation (6)

$\beta_{e}=\sqrt{3 \Omega_{\phi_{0}}\left(w_{0}+1\right)}$ 


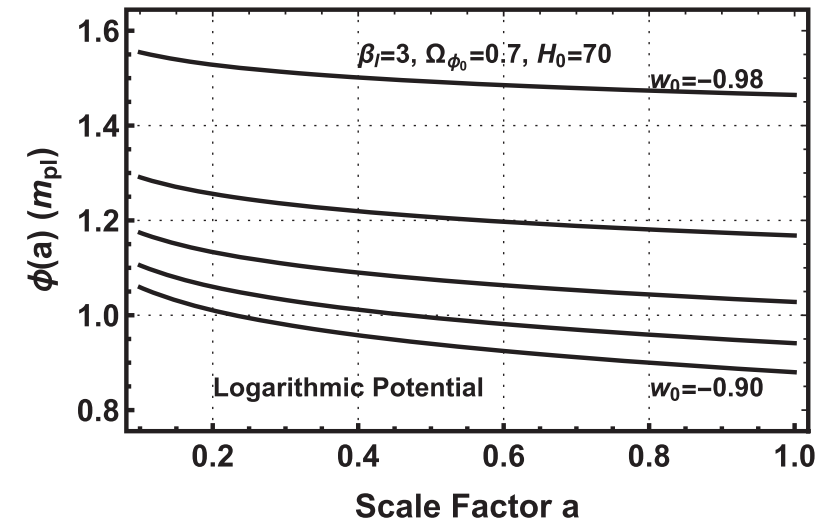

Figure 1. The evolution of the scalar field $\phi$ as a function of the scalar $a$ for the logarithmic beta function with $\beta_{l}=3.0$ for the five values of $w_{0}$ listed in the introduction.

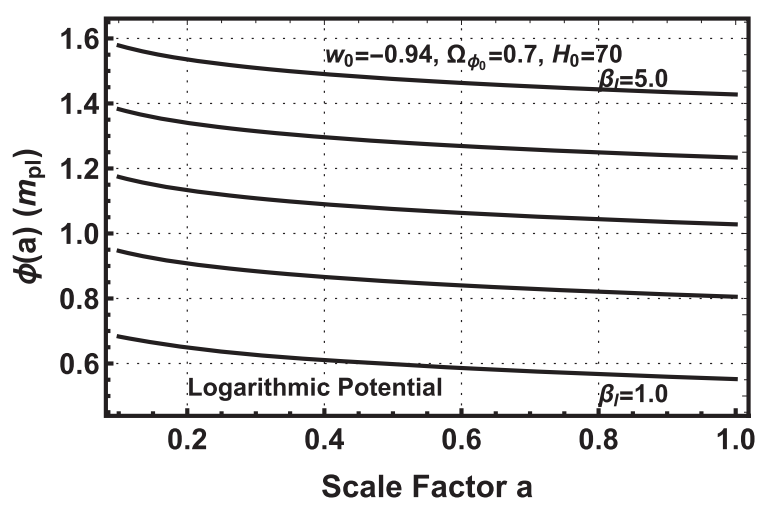

Figure 2. The evolution of the scalar field $\phi$ as a function of the scalar $a$ for the logarithmic beta function with the five values of $\beta_{l}$ and $w_{0}=-0.94$.

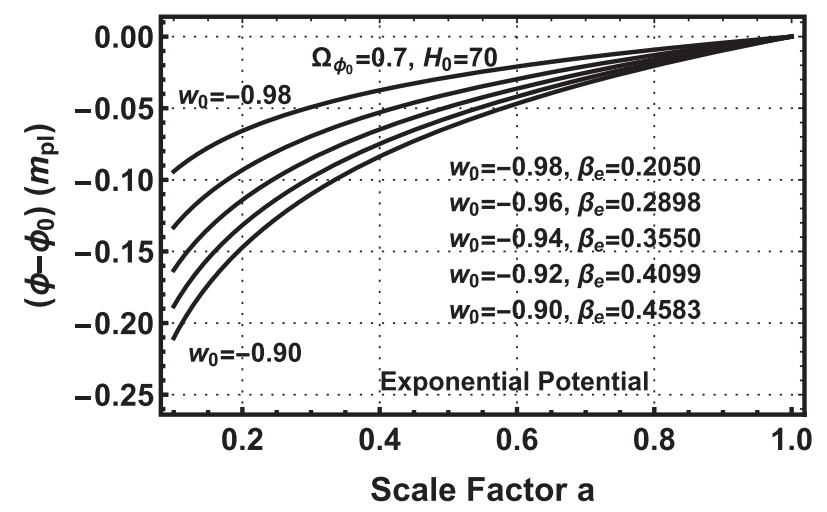

Figure 3. The evolution of the scalar field $\left(\phi-\phi_{0}\right)$ as a function of the scalar $a$ for the exponential beta function for the five values of $w_{0}$ listed in the introduction.

independent of $\phi$ or $\phi_{0}$, therefore there is no boundary condition to set $\phi_{0}$. The solutions for the relevant cosmological parameters and fundamental constants are all functions of $\left(\phi-\phi_{0}\right)$ therefore it is the appropriate parameter rather than the absolute values of $\phi$ and $\phi_{0}$. From the exponential potential beta function

$k\left(\phi-\phi_{0}\right)=\beta_{\mathrm{e}} \ln (a)$.

The evolution of $\left(\phi-\phi_{0}\right)$ is shown in Fig. 3. The values of $\beta_{\mathrm{e}}$ for

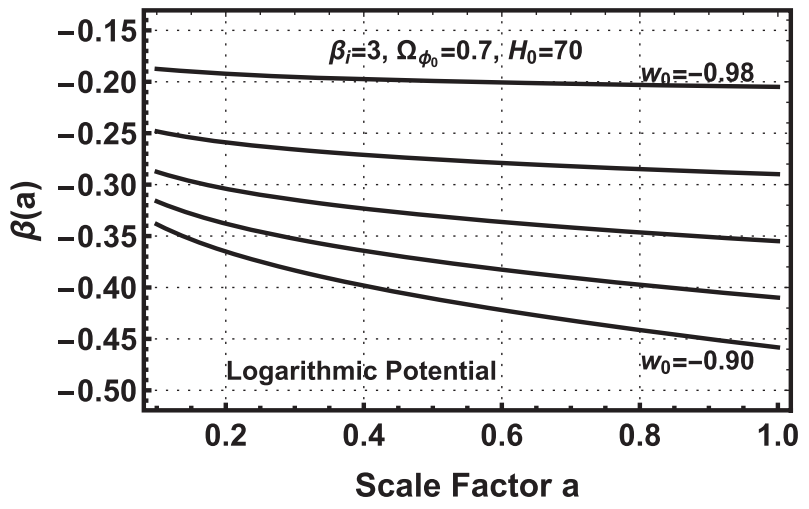

Figure 4. The evolution of $\beta(a)$ as a function of the scalar $a$ for the logarithmic potential with $\beta_{l}=3$ for the five values of $w_{0}$ listed in the introduction.

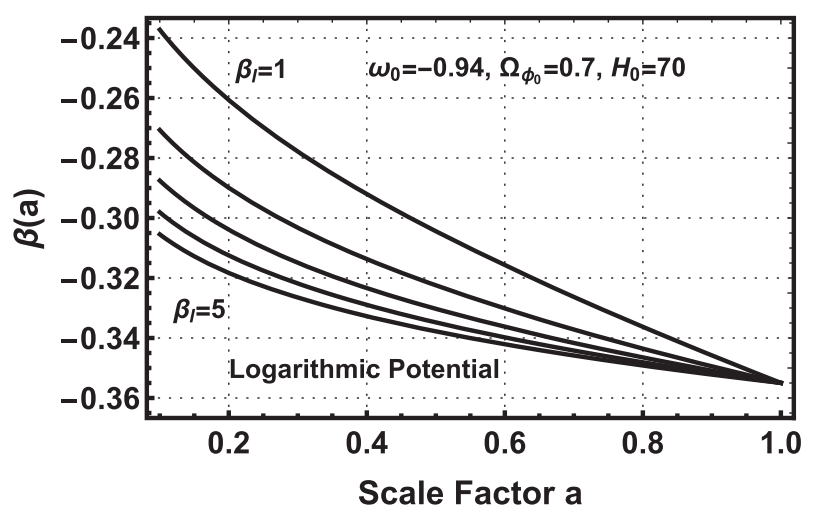

Figure 5. The evolution of $\beta(a)$ as a function of the scalar $a$ for the logarithmic potential with $w_{0}=-0.94$ for the five values of $\beta_{l}$ listed in the introduction.

the appropriate values of $w_{0}$ are listed in Fig. 3 and are all less than one.

An anonymous referee has pointed out that a constant beta function never reaches a fixed de Sitter point which requires a beta function value of zero. The referee also mentioned that for a small value of the beta function, as is found here, that space time is evolving towards a power-law geometry that might have interesting consequences in holography as discussed in Cicciarella, Mabillard \& Pieroni (2018).

\section{THE EVOLUTION OF THE BETA FUNCTION}

In the beta function formalism many of the cosmological parameters depend on the form of the beta function. Figs 4 and 5 display the evolution of the logarithmic potential beta functions for the five values of $w_{0}$ with $\beta_{l}=3$, (Fig. 4) and for the five values of $\beta_{i}$ with $w_{0}=-0.94$ (Fig. 5). The logarithmic beta functions are negative and between -0.1 and -0.5 for scale factors between 0.1 and 1 .

Fig. 6 shows the evolution of the exponential potential beta function for the five $\beta_{\mathrm{e}}, w_{0}$ pairs. The values are positive and constant which simplifies several of the subsequent calculations.

\section{THE POTENTIALS}

In the beta function formalism two different types of potentials play a prominent role. The first is the dark energy potential in the action $V(\phi)$ that does not depend on matter. The second, in analogy with 


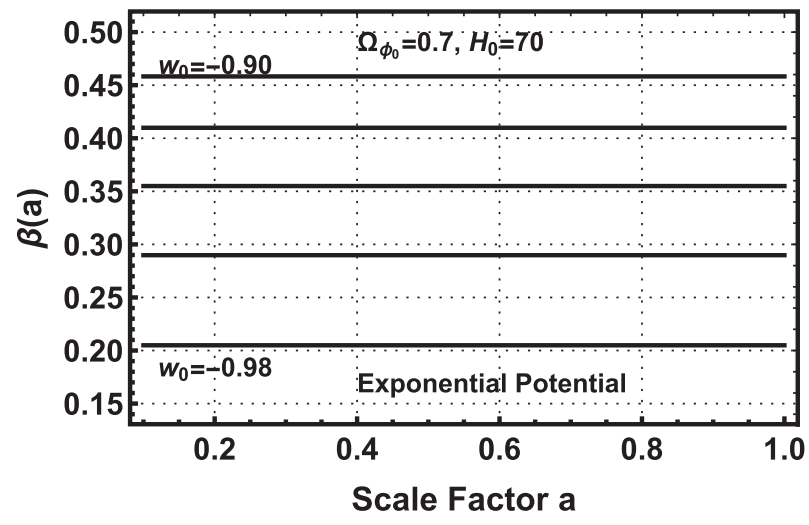

Figure 6. The evolution of $\beta(a)$ as a function of the scalar $a$ for the exponential potential.

particle physics, is termed the superpotential $W$ given by

$W(\phi)=-2 H(\phi)=-2 \frac{\dot{a}}{a}$.

Even though the Hubble parameter $H$ is the parameter of interest $W$ is utilized here to be consistent with the literature on beta functions. Both the dark energy potential $V(\phi)$ and the superpotential $W(\phi)$ can be expressed in terms of $\beta(\phi)$ (Cicciarella \& Pieroni 2017) by

$W(\phi)=W_{0} \exp \left\{-\frac{1}{2} \int_{\phi_{0}}^{\phi} \beta\left(k \phi^{\prime}\right) k \mathrm{~d} \phi^{\prime}\right\}$

and

$V(\phi)=\frac{3}{4 k^{2}} W_{0}^{2} \exp \left\{-\int_{\phi_{0}}^{\phi} \beta\left(k \phi^{\prime}\right) k \mathrm{~d} \phi^{\prime}\right\}\left(1-\frac{\beta^{2}(k \phi)}{6}\right)$,

where $W_{0}$ is the current value of $W$ equal to $-2 H_{0}$. Note that the superpotential is always denoted as a capital $W$ and the dark energy equation of state by a lower case $w$. The potential in equation (20) is referred to as the beta potential of the model potential. It differs from the model potential by the factor of $\left(1-\frac{\beta^{2}(\phi)}{6}\right)$. As long as this factor is close to one the beta potential is an accurate, but not exact, representation of the model potential.

\subsection{The logarithmic potential}

The model logarithmic potential is given by

$V_{m}(\phi)=\frac{3}{4 k^{2}} W_{0}^{2}\left(\frac{\ln (k \phi)}{\ln \left(k \phi_{0}\right)}\right)^{\beta_{l}}$

with the beta function shown in equation (8). The logarithmic beta potential is given by

$V_{b}(\phi)=\frac{3}{4 k^{2}} W_{0}^{2}\left(\frac{\ln (k \phi)}{\ln \left(k \phi_{0}\right)}\right)^{\beta_{l}}\left(1-\frac{\beta_{l}^{2}}{6(k \phi \ln (k \phi))^{2}}\right)$.

The logarithmic potential is decreasing as the scale factor increases. Fig. 7 shows the potential with $\beta_{l}$ fixed at 3 for the five different values of $w_{0}$. The solid lines in Fig. 7 show the beta potential which follows the model potential (dashed) quite well, particularly for values of $w_{0}$ close to minus one. The accuracy of the fit is quantified in Section 6.4 for all of the potentials.

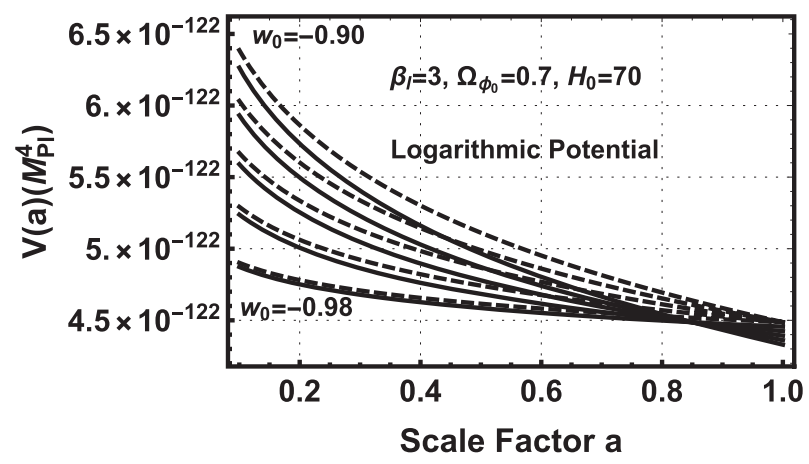

Figure 7. The evolution of the model logarithmic potential with $\beta_{l}=3$ is shown by the dashed lines and the solid lines indicate the evolution of the beta logarithmic potential.

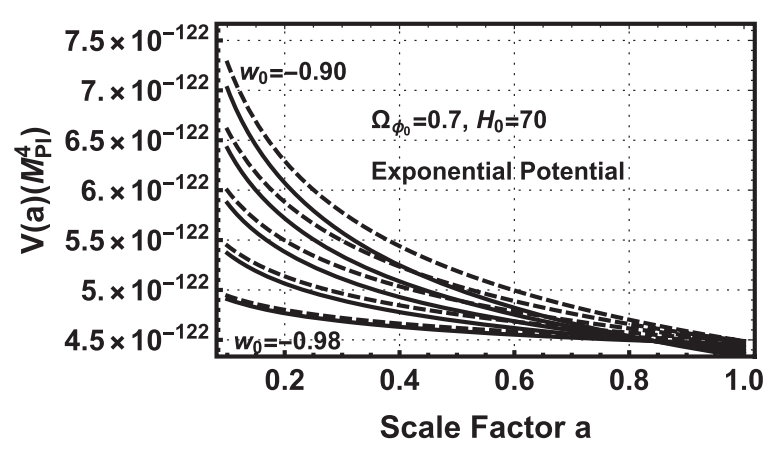

Figure 8. The evolution of the model exponential potential is shown by the dashed lines and the solid lines indicate the evolution of the beta exponential potential.

\subsection{The exponential potential}

The model potential is of the form

$V_{\mathrm{m}}(\phi)=\frac{3}{4 k^{2}} W_{0}^{2} \exp \left(-\beta_{\mathrm{e}} k\left(\phi-\phi_{0}\right)\right)$

with a beta potential of

$V_{\mathrm{b}}(\phi)=\frac{3}{4 k^{2}} W_{0}^{2} \exp \left(-\beta_{\mathrm{e}} k\left(\phi-\phi_{0}\right)\right)\left(1-\frac{\beta_{\mathrm{e}}^{2}}{6}\right)$.

Fig. 8 shows the evolution of exponential model and beta potentials.

\subsection{Normalization}

It is clear that the beta dark energy potentials have the desired model potentials multiplied by $\left(1-\frac{\beta(\phi)^{2}}{6}\right)$ which produces both an offset and a deviation from the model potentials. The deviation is expected to be small since $\frac{\beta(\phi)^{2}}{6}$ is much less than one in most cases. In paper I the potential was normalized to be $\frac{3}{4} W_{0}^{2}$ at a scale factor of one producing a potential slightly different than the true beta potential. In this work that practice has been abandoned and no normalization has been applied. As a result the beta potentials shown in Figs 7 and 8 cross over each other at $a \approx 0.8$ due to the $\frac{\beta(\phi)^{2}}{6}$ term.

\subsection{Accuracy of fit}

The cosmological parameters derived by the beta function formalism are only useful if the beta potentials accurately represent the model potentials. Figs 9 and 10 show the fractional deviation of the 


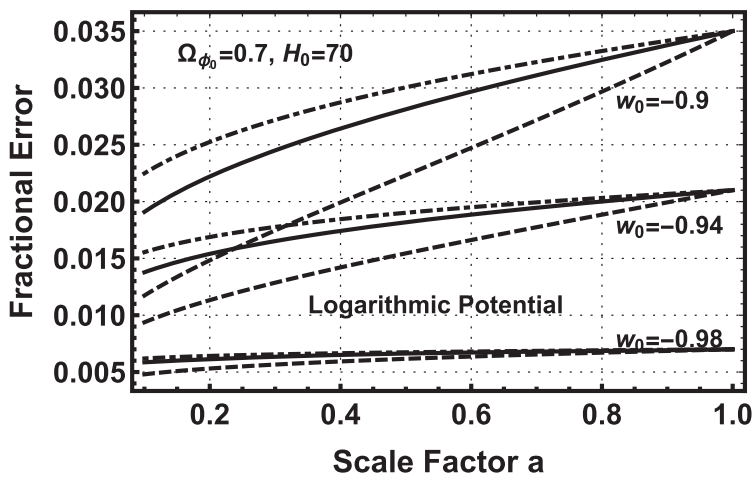

Figure 9. The fractional deviation of the beta logarithmic law potentials from the model potentials with $\beta_{l}=1$, dashed lines, $\beta_{l}=3.0$, solid lines, and $\beta_{p}=5.0$, dot-dashed lines. For each $\beta_{l}$ the tracks are marked with the value $w_{0}$ at the end.

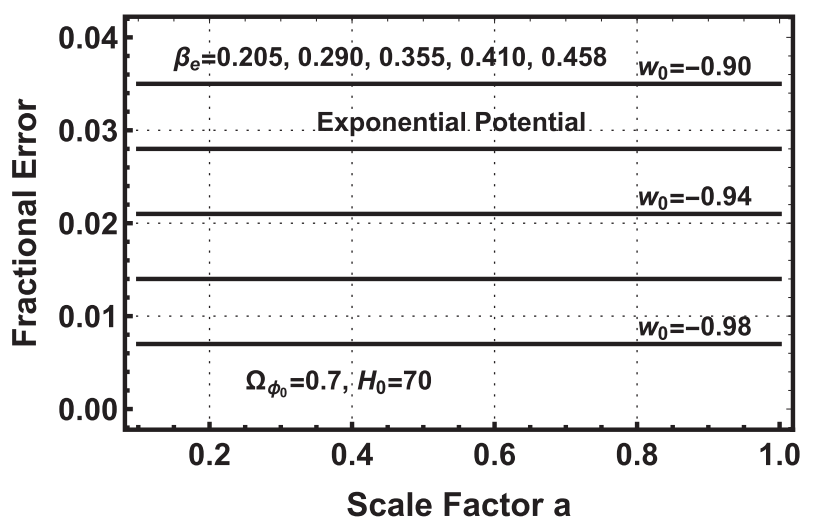

Figure 10. The same as for Fig. 9 except for the exponential-law potentials. All five values of $\beta_{\mathrm{e}}$ are shown with the values of $w_{0}$ marked on the figure.

beta potentials from the model potentials to quantify the deviations of the beta potentials from the model potentials. For the logarithmic potential the minimum, median, and maximum $\beta_{l}$ values are shown with $w_{0}$ values equal to $-0.98,-0.94$, and -0.9 to show the extremes without excessive overlap of tracks in the figures. For the exponential potential all of the cases are shown since they do not overlap. In paper I a conservative limit of only accepting solutions with fractional deviations of 1 per cent or less was adopted. In this paper that limit is expanded to 4 per cent which is a higher accuracy than the accuracy of most of the available observational data.

\subsubsection{The logarithmic beta potential fractional error}

The primary feature of the logarithmic potential fractional deviation in Fig. 9 is that all of the cases are within the acceptable error of 0.04. Unlike the normalized cases of paper I the highest fractional deviation for the logarithmic beta potential is at a scale factor of one increasing for values of $w_{0}$ further from minus one but independent of the value of $\beta_{l}$. The evolution away from $a=1$ is dependent on $\beta_{l}$ but is decreasing for lower values of $a$. All of the logarithmic cases are therefore retained in the subsequent analysis.

\subsubsection{The exponential beta potential fractional errors}

The exponential beta potential fractional errors shown in Fig. 10 are set by the values of $w_{0}$ which also sets the value of $\beta_{\mathrm{e}}$. As expected the fractional deviations of the exponential beta potential are independent of the scale factor since $\beta(\phi)$ is constant for a given $w_{0}$ and all fall in the acceptable range. As with the logarithmic beta potential all of the exponential cases are retained in the subsequent analysis.

\section{THE MAT TER DENSITY}

The dark energy potentials are independent of matter but both baryonic and dark matter must be taken into account to calculate accurate analytic solutions for fundamental constants and cosmological parameters. Matter is represented by the $S_{\mathrm{m}}$ term in the action, equation (3). From Cicciarella \& Pieroni (2017) and paper I the matter density as a function of the scalar is given by

$\rho_{\mathrm{m}}(\phi)=\rho_{\mathrm{m}_{0}} \exp \left(-3 \int_{\phi_{0}}^{\phi} \frac{\mathrm{d} \phi^{\prime}}{\beta\left(\phi^{\prime}\right)}\right)$,

where $\rho_{m_{0}}$ is the present-day mass density. Different beta functions produce different functions for $\rho_{\mathrm{m}}$ as a function of $\phi$ hiding the universality of the matter density when expressed as a function of the scale factor $a$

$\rho_{\mathrm{m}}(a)=\rho_{\mathrm{m}_{0}} \exp \left(-3 \int_{1}^{a} \mathrm{~d} \ln \left(a^{\prime}\right)\right)=\rho_{m 0} a^{-3}$

as expected, independent of $\beta(\phi)$.

\section{THE SUPERPOTENTIAL W AND THE HUBBLE PARAMETER H}

From equation (18) it is obvious that calculating the superpotential $W$ is equivalent to calculating the Hubble Parameter $H$. As shown in Cicciarella \& Pieroni (2017) and paper I the differential equation for $W$ with matter is

$W W_{, \phi}+\frac{1}{2} \beta W^{2}=-2 \frac{\rho_{m}}{\beta}$,

where the notation, ${ }_{\phi}$ indicates the derivative with respect to the scalar $\phi$. Paper I includes two specific examples, the power and inverse power-law potentials and their related beta functions. Here a more general solution is presented that gives a better insight of the process. The solutions to equation (27) utilize integrating factors $f(x)$, where $x=k \phi$ for ease of notation. The integrating factors multiply both sides of equation (27) to create an exact equation that can be integrated. The exact form on the left of the equation has the form of the left side of equation (28). The right side is then integrated to provide the solution for $W$ :

$\frac{\mathrm{d}}{\mathrm{d} x}\left(\frac{1}{2} W^{2}(x) f(x)\right)=-2 f(x) \frac{\rho_{\mathrm{m}}(x)}{\beta(x)}$.

Comparison with equation (27) shows that the integrating factor must satisfy

$\frac{\mathrm{d} f(x)}{\mathrm{d} x}=\beta(x) f(x)$

which determines $f(x)$. Writing the equation out as the equality of two differentials gives

$\mathrm{d}\left(W^{2}(x) f(x)\right)=-4 f(x) \frac{\rho_{m}(x)}{\beta(x)} \mathrm{d} x$.

Integrating both sides of equation (30) gives

$W^{2}(x) f(x)-W_{0}^{2} f\left(x_{0}\right)=-4 \int_{x_{0}}^{x} f(x) \frac{\rho_{m}(x)}{\beta(x)} \mathrm{d} x$. 
Equation (31) can be solved as a function of $x$ or the much more useful function of $a$ using $x(a)$ from equations (15) and (17) and the much simpler $\rho_{m}(a)$ from equation (26). The beta function provides the conversion of $\mathrm{d} x$ on the right-hand side of equation (30) to $\mathrm{d} a$

$\mathrm{d} x=\beta(x(a)) \mathrm{d} \ln (a)=\beta(x(a)) \frac{\mathrm{d} a}{a}$.

The beta function in equation (32) cancels the beta function on the right-hand side of equation (30). The right-hand side of equation (30) is now a function of $a$ rather than $x$ and the integral of the right-hand side is

$-4 \rho_{m_{0}} \int_{1}^{a} f\left(x\left(a^{\prime}\right)\right) a^{\prime-4} \mathrm{~d} a^{\prime}$.

After integrating the left side of equation (30) and re-arranging the final answer is

$W(a)=-\left\{-4 \frac{\rho m_{0}}{f(x(a))} \int_{1}^{a} f\left(x\left(a^{\prime}\right)\right) a^{\prime-4} \mathrm{~d} a+W_{0}^{2} \frac{f(x(a=1)}{f(x(a))}\right\}^{\frac{1}{2}}$.

The integrating factors for the logarithmic and exponential potentials are

$$
\begin{array}{rc}
(\ln (k \phi))^{-\beta_{l}} & \text { logarithmic } \\
\exp \left[\beta_{\mathrm{e}} k\left(\phi-\phi_{0}\right)\right] & \text { exponential. }
\end{array}
$$

The integral in equation (33) for the exponential integrating factor is quite simple and analytic. The integral for the logarithmic integrating factor is not analytic and must be done numerically since it contains the PL function for $x(a)$ given in equation (13).

$$
\begin{aligned}
W(a)= & -\left[-4 \rho_{m_{0}}\left(\operatorname { l n } ( k \phi ( a ) ) ^ { \beta _ { l } } \int _ { 1 } ^ { a } \left(\ln \left(k \phi\left(a^{\prime}\right)\right)^{-\beta_{l}} a^{\prime-4} \mathrm{~d} a^{\prime}\right.\right.\right. \\
& \left.+W_{0}^{2}\left(\frac{\ln (k \phi(a))}{\ln \left(k \phi_{0}\right)}\right)^{\beta_{l}}\right]^{\frac{1}{2}} \log \\
W(a)= & -\left[\frac{-4 \rho_{m_{0}}}{\beta_{e}^{2}-3}\left(a^{-3}-a^{-\beta_{e}^{2}}\right)+W_{0}^{2} a^{-\beta_{e}^{2}}\right]^{\frac{1}{2}} \exp ,
\end{aligned}
$$

where $k \phi(a)$ is given by equation (13) for the logarithmic potentials. The superpotential is a negative quantity therefore the negative solution of the square roots in equations (36) are used.

\subsection{The Hubble parameter as a function of the scale factor}

The Hubble parameter is simply $-\frac{W(a)}{2}$. As was found in paper I for the power and inverse power-law potentials the evolution of the Hubble parameter for the logarithmic and exponential potentials is indistinguishable from the $\Lambda \mathrm{CDM}$ evolution at the scale of the plots. To highlight the true differences Fig. 11 shows the ratio of the Hubble parameter for logarithmic potential to the $\Lambda$ CDM minus one as a function of the scale factor. In Fig. $11 \beta_{l}$ is held constant at three and each of the five values of $w_{0}$ are plotted. The same ratio is plotted for the five exponential potential cases in Fig. 12. In both the logarithmic and exponential cases the deviation from the $\Lambda \mathrm{CDM}$ case is small and peaks at $a \approx 0.5$ as expected. The similarity of the Hubble parameter evolution for a dynamic quintessence cosmology to the static $\Lambda \mathrm{CDM}$ cosmology makes it a poor discriminator between the two cases. One percent accuracy observations of $H(a)$ at redshifts near one are required to distinguish between the two. The reason for the similarity of the evolutions is given in the next section.

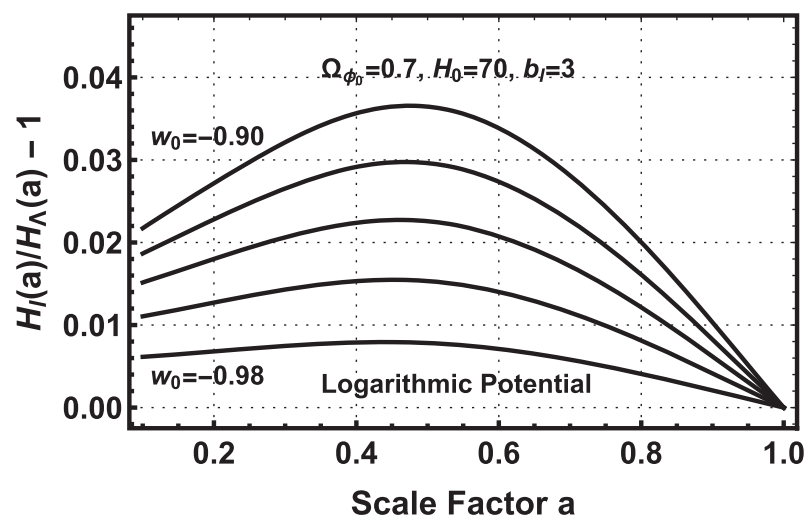

Figure 11. The ratio of the logarithmic potential evolution of the Hubble parameter $H_{l}(a) . \beta_{l}$ is held constant at 3 and all five of the $w_{0}$ values are plotted.

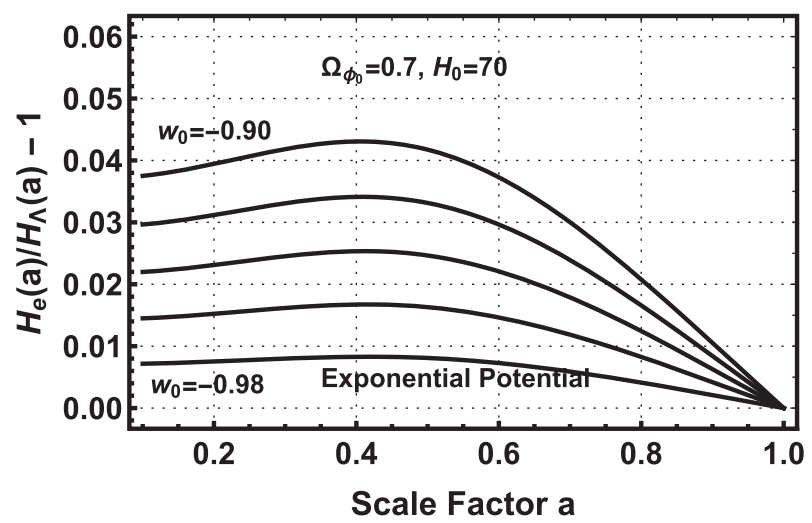

Figure 12. The same as in Fig. 11 except for the five exponential potential cases.

\subsection{The evolution of the dark energy density}

From the Einstein equation with mass

$3 H^{2}=\rho_{m}+\rho_{\phi}$

it is clear that

$\rho_{\phi}=3 H^{2}-\rho_{m}=3 H^{2}(a)-\frac{\rho_{m_{0}}}{a^{3}}$

for a flat universe. Figs 13 and 14 show the evolution of the dark energy density for the logarithmic and exponential potentials, respectively. The dashed line in the figures shows the evolution of the matter density. The reason for the similarity of the quintessence evolution of $H(a)$ to the $\Lambda \mathrm{CDM}$ evolution is shown in the figures. The quintessence dark energy density evolves very slowly in the current dark energy dominated epoch, mimicking the static cosmological constant dark energy density. The quintessence dark energy density only evolves significantly at high redshift in the matter dominated era. This is why the $H(a)$ evolution is essentially similar for the two cosmologies and may be true for most freezing cosmologies. There are thawing quintessence cosmologies (Scherrer \& Sen 2008) however their potentials are extremely flat and must match the same value of $H_{0}$ as the freezing models.

\section{THE DARK ENERGY EQUATION OF STATE}

A primary observational indicator of a dynamical cosmology is a dark energy equation of state different from the cosmological 


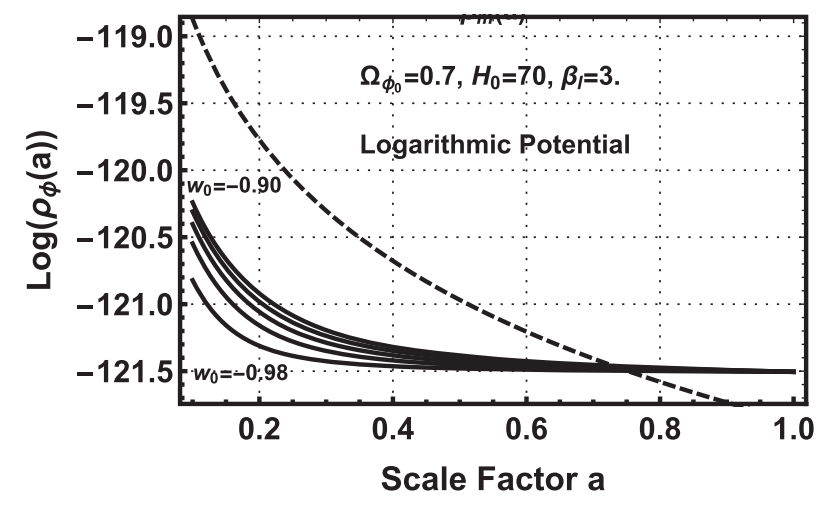

Figure 13. The $\log _{10}$ of the dark energy density values as a function of the scale factor for the logarithmic potential. The dashed line is the matter density which decreases below the dark energy density near a scale factor of 0.75 .

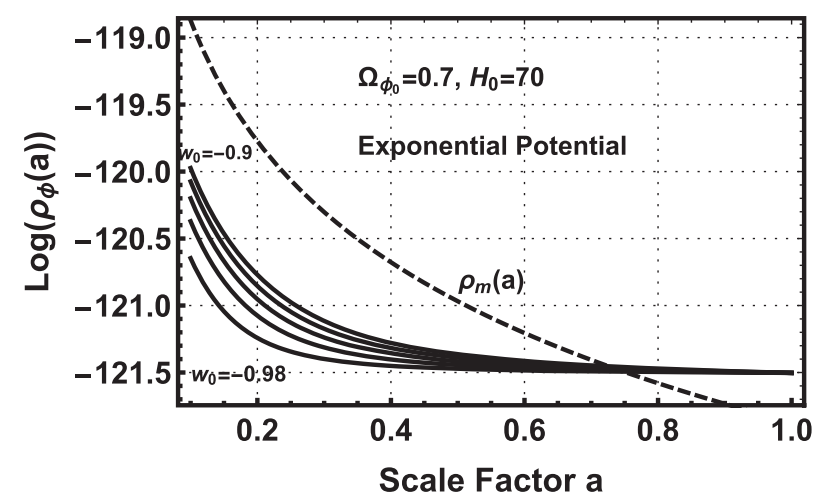

Figure 14. The dark energy density values as a function of the scale factor for the exponential potential. As in Fig. 13 the dashed line shows the matter density.

constant value of minus one. From paper I

$$
\begin{aligned}
1+w(\phi) & =\frac{k^{2} \beta^{2}}{3}\left(1-\frac{4 \rho_{m_{0}} a^{-3}}{3 W^{2}}\right)^{-1} \\
& =\frac{k^{2} \beta^{2}}{3}\left(1-\Omega_{m}\right)^{-1}=\frac{k^{2} \beta^{2}(\phi)}{3 \Omega_{\phi}}
\end{aligned}
$$

for a flat universe. Fig. 15 shows the evolution of $(w(a)+1)$ for the logarithmic dark energy potential with $\beta_{l}=3$ and all five values of $w_{0}$. Fig. 16 shows the evolution of $(w(a)+1)$ for the exponential dark energy potential with the $\beta_{\mathrm{e}}$ values set by $\left(w_{0}+1\right)=0.02$, $0.04,0.06,0.08$, and 0.1 .

A common feature of all of the potentials in this paper and paper I is a very slow late time, $a>0.5$ evolution of $w(a)$ with significant evolution for scale factors between 0.1 and 0.5 . This indicates that at least for the quintessence cosmology that high-redshift observations have the best chance of detecting the presence of dynamical dark energy. The shapes of the logarithmic and exponential potential $w(a)$ are quite similar, particularly for the lower values of $w_{0}$, while they are more divergent for the higher values. Any determination of the dark energy potential from the $w(a)$ tracks would require a secure knowledge of $w_{0}$ and very accurate measurements of $w(a)$ at higher redshifts. The required level of accuracy is beyond current observational capabilities. Detection of the predicted value of $w(a) \approx-0.5$ at $a=0.2, z=4$, however, might be possible with

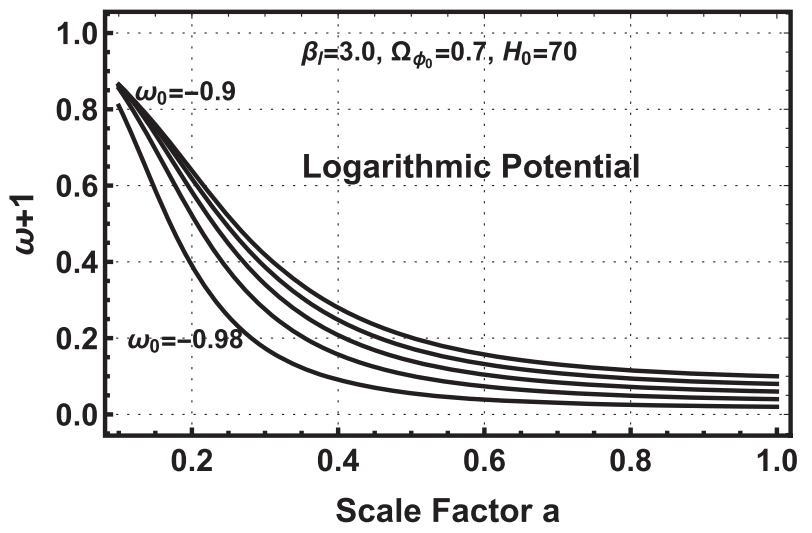

Figure 15. The evolution of $(w(a)+1)$ as a function of $a$ for the logarithmic dark energy potential with $\beta_{l}=3$ and all five values of $w_{0}$.

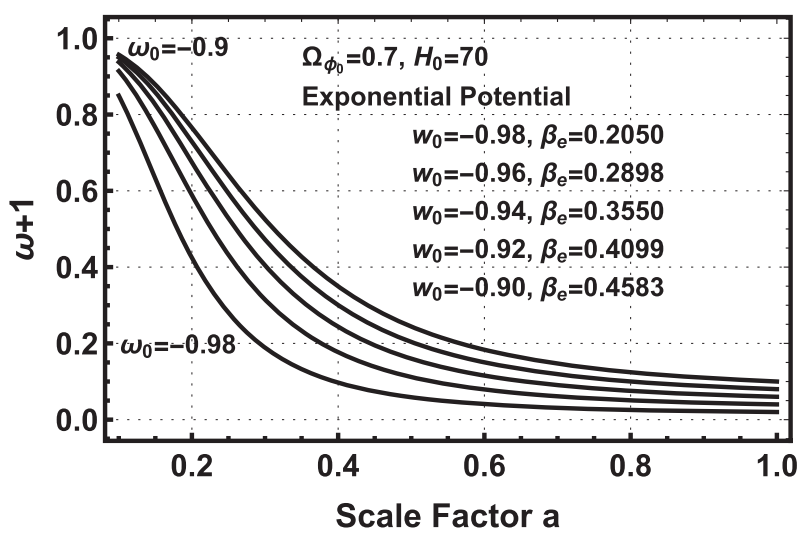

Figure 16. The evolution of $(w(a)+1)$ as a function of $a$ for the exponential dark energy potential for the five $\beta_{e}$ values set by $\left(w_{0}+1\right)=0.02,0.04$, $0.06,0.08$, and 0.1 .

present techniques. Further discussion of $w(a)$ observations occurs in Section 11.1.

\subsection{The fundamental constants}

Paper I gives an extensive discussion of the evolution of the fundamental constants for both the proton to electron mass ratio $\mu$ and the fine structure constant $\alpha$ in terms of a change of $\phi$ and a coupling constant $\zeta_{c}$ where $c$ is $\mu$ or $\alpha$ (Nunes \& Lidsey 2004).

$\frac{\Delta c}{c}=\zeta_{c} k\left(\phi-\phi_{0}\right)=\zeta_{c} \int_{1}^{a} \beta\left(a^{\prime}\right) \mathrm{d} \ln a^{\prime}, \quad c=\alpha, \mu$.

The first equality is usually interpreted as the first term of a Taylor expansion of a possibly more complicated coupling. The observational constraints on $\Delta \alpha / \alpha$ and $\Delta \mu / \mu$ are of the order $10^{-6}$ or less, justifying the assumption. The last equality, not shown in paper I, explicitly shows the connection between the beta function and the evolution of the fundamental constants. Sections 4.1 and 4.2 show the transformation of $\beta(\phi)$ to $\beta(a)$ via the formulae for $\phi(a)$.

Fig. 17 shows the evolution of $\Delta \mu / \mu$ versus the scale factor for the logarithmic potential with $\beta_{l}=3$ and the five values of $w_{0}$. The positive and negative evolutions simply indicate that the coupling could have either a positive or negative sign. The coupling is arbitrarily set to $\pm 10^{-6}$ for the figure. The evolution of the fine structure constant is identical for the same coupling constant. The evolution reflects the evolution of $\phi(a)$ since the coupling is assumed 


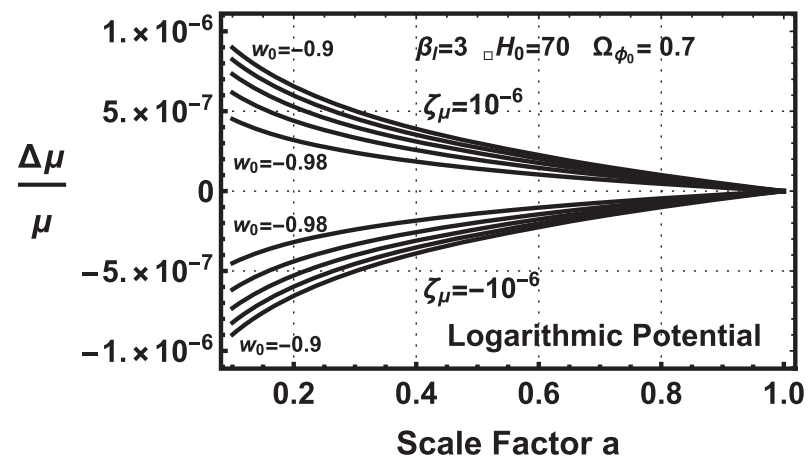

Figure 17. The evolution of $\frac{\Delta \mu}{\mu}$ for the logarithmic dark energy potential with $\beta_{\mathrm{e}}=3$ and $w_{0}=-0.98,-0.96,-0.94,-0.92$, and -0.98 . The coupling constant $\zeta_{\mu}$ is set to $\pm 10^{-6}$ as an example.

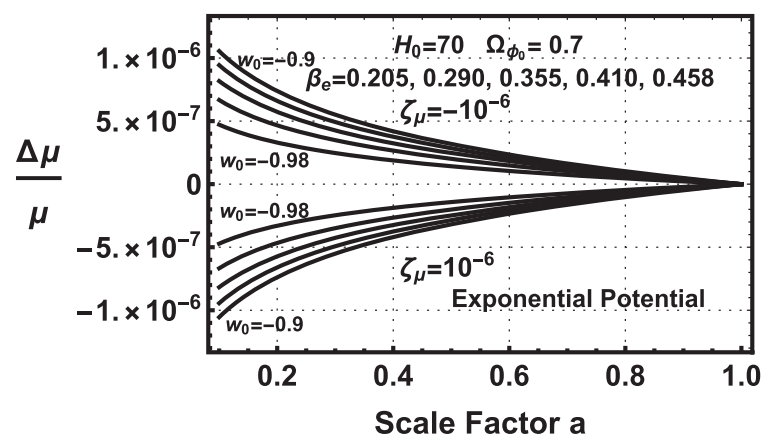

Figure 18. The evolution of $\frac{\Delta \mu}{\mu}$ for the exponential dark energy potential for the $\beta_{\mathrm{e}}$ values set by $\left(w_{0}+1\right)=0.02,0.04,0.06,0.08$, and 0.1 . The coupling constant $\zeta_{\mu}$ is set to $\pm 10^{-6}$.

to be a constant. As expected the higher the deviation of $w_{0}$ is from minus one the larger the evolution of $\mu$. Similar to the power-law potentials in paper I the evolution of $\Delta \mu / \mu$ relatively insensitive to changes in $\beta_{l}$.

Fig. 18 shows the evolution of $\mu$ for the exponential potential. As described in Section $4.2 w_{0}$ and $\beta_{\mathrm{e}}$ are not independent variables in the exponential case. In Fig. 18 the five values of $w_{0}$ are retained as in Fig. 3 with the appropriate values of $\beta_{\mathrm{e}}$ for each case. The values of $\beta_{\mathrm{e}}$ are shown in Fig. 18.

\subsubsection{Observational constraints on $\frac{\Delta \mu}{\mu}$}

As discussed in paper I the primary constraint on a variation of $\mu$ is $\Delta \mu / \mu \leq \pm 10^{-7}$ from Bagdonaite et al. (2013) and Kanekar et al. (2015) at a redshift of 0.88582 . This measurement defines an allowed and a forbidden parameter space in the $\zeta_{\mu} w_{0}$ plane. The first parameter, $\zeta_{\mu}$, defines the limits on the allowed deviation from the standard model, $\zeta_{\mu}=0$, and the second, $w_{0}$, the allowed deviation from the cosmological constant, $\left(w_{0}+1\right)=0$. The upper limit on $\zeta_{\mu}$ is given by

$\zeta_{\mu}=\frac{\Delta \mu / \mu}{\int_{1}^{a_{o b}} \beta\left(a^{\prime}\right) \mathrm{d} \ln \left(a^{\prime}\right)}=\frac{\Delta \mu / \mu}{\sqrt{3 \Omega_{0}\left(w_{0}+1\right)} \ln \left(a_{\mathrm{ob}}\right)}$,

where $a_{\mathrm{ob}}$ is the scale factor at the epoch of the observation. The second equality shows explicitly the dependence on $w_{0}$. Fig. 19 shows the allowed and forbidden parameter space for the logarithmic dark energy potential and Fig. 20 the parameter spaces for the exponential potential.

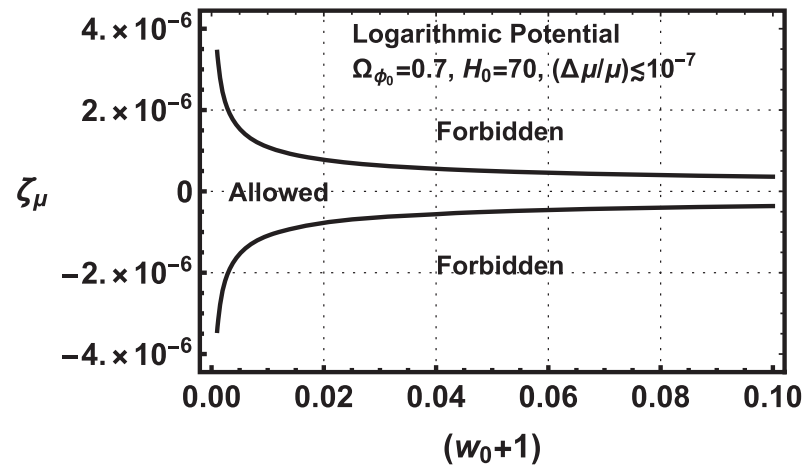

Figure 19. The allowed and forbidden parameter spaces in the $\zeta_{\mu}-w_{0}$ plane for the logarithmic dark energy potential.

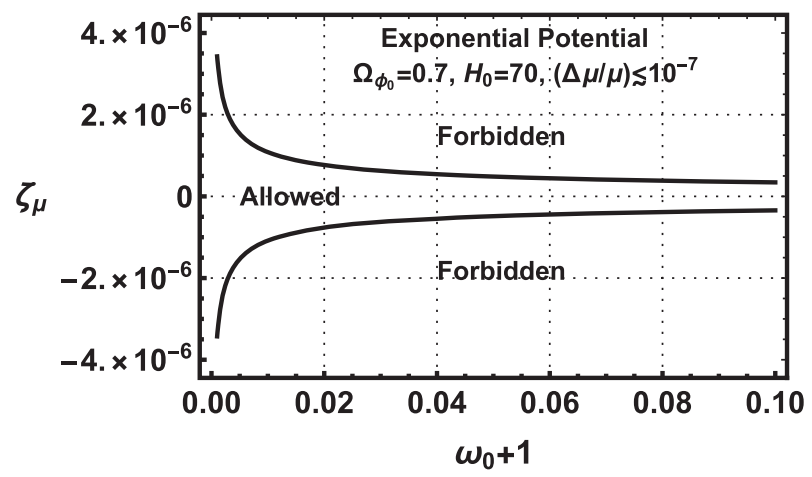

Figure 20. The allowed and forbidden parameter spaces in the $\zeta_{\mu}-w_{0}$ plane for the exponential dark energy potential.

The plots start at $\left(w_{0}+1\right)=0.001$ to avoid the plus and minus infinite values of $\zeta_{\mu}$ at $\left(w_{0}+1\right)=0$. The allowed parameter space contains the $\Lambda \mathrm{CDM}$ cosmology which is the 0,0 point in the plots. Although constrained to either small values of $\zeta_{\mu}$ or $\left(w_{0}+1\right)$ there is still room in the allowed parameter space to accommodate quintessence.

\section{RELEVANT BUT NOT DIRECTLY OBSERVABLE PARAMETERS}

There are several cosmological parameters that are relevant but not directly observable. Here two parameters, the time derivative of the scalar field and the dark energy pressure, are calculated as functions of the scale factor $a$.

\subsection{The evolution of the time derivative of the scalar}

As shown in paper I the time derivative of the scalar $\dot{\phi}$ is simply the Hubble parameter times the beta function.

$k \dot{\phi}=a \frac{k \mathrm{~d} \phi}{\mathrm{d} a} \frac{\dot{a}}{a}=\beta H$

Fig. 21 shows the evolution of $\dot{\phi}$ with respect to the scale factor $a$ for the logarithmic dark energy potential for the five values of $w_{0}$ with $\beta_{l}$ held constant at three. The differences in the tracks are entirely due the differences in the beta function since the values of $H(a)$ are essentially invariant with respect to the input parameters as shown in Section 8.1 and paper I. The values of $\dot{\phi}$ are negative because the logarithmic beta function is negative.

Fig. 22 shows the tracks of $\dot{\phi}$ as a function of $a$ for the exponential 


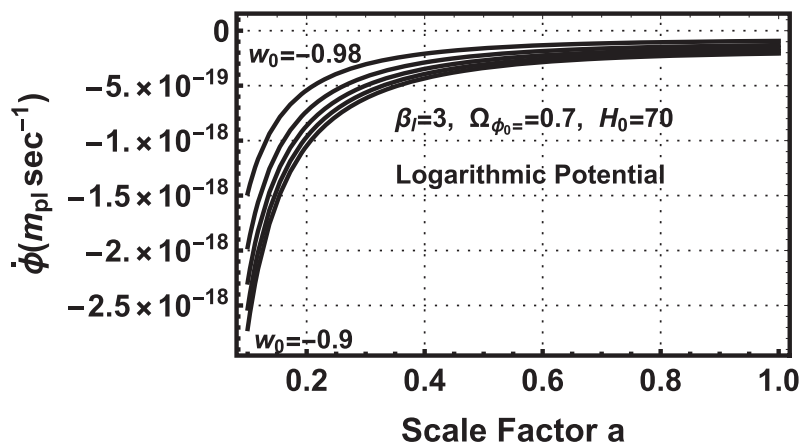

Figure 21. The time derivative of the scalar for the logarithmic potential for the five values of $w_{0}$ with $\beta_{l}$ held constant at three.

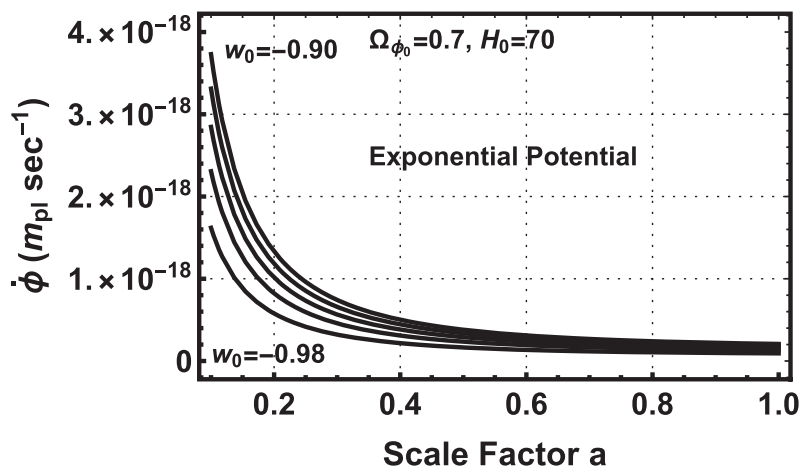

Figure 22. The time derivative of the scalar for the exponential potential for the five values of $w_{0}$ and their associated values of $\beta_{\mathrm{e}}$.

dark energy potential for the five values of $w_{0}$ and the $\beta_{\mathrm{e}}$ values associated with them. Both the logarithmic and the exponential have $\dot{\phi}$ values approaching zero at the present time.

\subsection{The evolution of the dark energy pressure}

The dark energy pressure comes from the second of the Einstein equations. $-2 \dot{H}=\rho_{m}+\rho_{\phi}+p_{\phi}$ where $\dot{H}=-\frac{1}{2} \dot{\phi} W_{, \phi}$. From equation (27)

$W_{, \phi}=-\frac{2 \rho_{m}}{\beta W}-\frac{1}{2} \beta W$

which yields using $k \dot{\phi}=-\frac{\beta W}{2}$

$p_{\phi}=-2 \rho_{m}-\frac{k^{2}}{2} \dot{\phi}^{2}+3 H^{2}$.

Fig. 23 shows the evolution of the dark energy pressure for the logarithmic dark energy potential for the five values of $w_{0}$ with $\beta_{l}=3$. Since the pressure is negative the negative numbers rather than the logarithms are plotted. As expected from the dark energy density plots the $p_{\phi}$ tracks cross over themselves. Fig. 24 shows the $p_{\phi}$ for the exponential potential for the five $\beta_{\mathrm{e}}, w_{0}$ pairs.

\section{RELEVANCE OF THE ANALYSIS}

This paper completes the investigation started in paper I of four common dark energy potentials in a quintessence cosmology. Here the relevance of the findings to important cosmological and new physics questions is examined. The literature on determining cosmological parameters based on observations is vast and it is not the

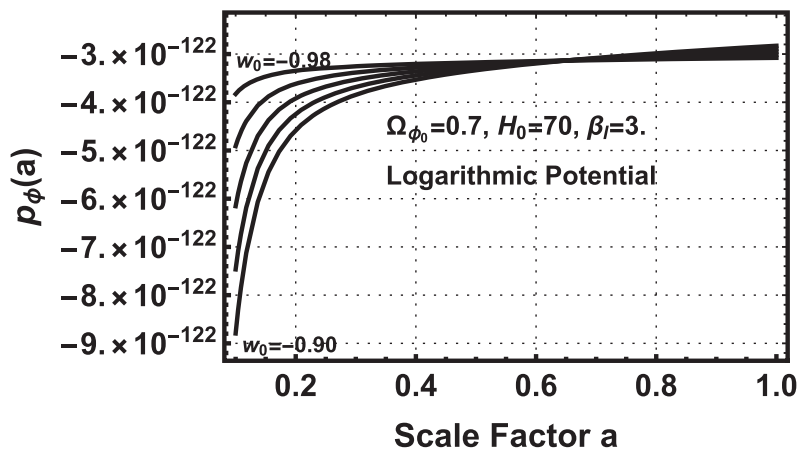

Figure 23. The dark energy pressure for the five values of $w_{0}$ with a logarithmic potential with $\beta_{l}=3$.

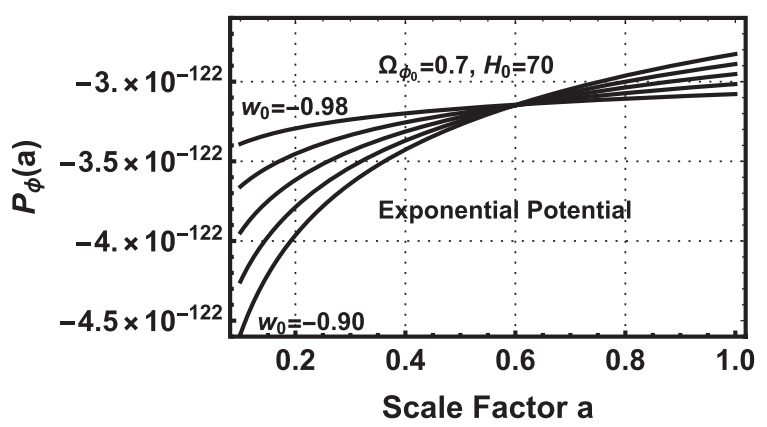

Figure 24. The dark energy pressure for the five $\beta_{\mathrm{e}}, w_{0}$ pairs with an exponential potential.

purpose of this section to determine the veracity of the various studies. Instead the following points out which parameters calculated in this study and paper I are relevant to the important questions and how they may differ from the current body of work.

\subsection{Dynamical versus static dark energy}

What observations can discriminate between a dynamic dark energy quintessence cosmology and a static dark energy $\Lambda$ CDM Universe? An important finding is that due to the flatness of the quintessence potentials in the dark energy dominated eras both cosmologies predict essentially identical evolution of the Hubble parameter $H(a)$. $H(a)$ measurements, therefore, cannot effectively discriminate between the two cases. Measurements that differed from the predicted evolution would, however, rule out both cosmologies.

Measurements of the dark energy equation of state $w(a)$ and the values of the fundamental constants $\mu$ and $\alpha$ can discriminate between dynamical and static dark energy. A confirmed observation of $w(a) \neq-1$ or a change in the value of a fundamental constant would rule out $\Lambda \mathrm{CDM}$ but would be consistent with quintessence or other dynamical dark energy cosmologies. Tests for a value of $w(a) \neq-1$ eg. Avsajanishvilli et al. (2017), are often conducted using the Chevallier-Polarsky-Linder (CPL) linear model (Chevallier \& Polarski 2001; Linder 2003).

$w(a)=w_{0}+w_{a}(1-a)$.

Examination of Figs 15 and 16 indicates that the model is a reasonable fit at low redshifts but is a bad fit at high redshifts where $w(a)$ is evolving rapidly in the quintessence cosmology. The tracks in these figures provide more realistic templates to compare with observations than the CPL linear model. The shapes of the $w(a)$ tracks 


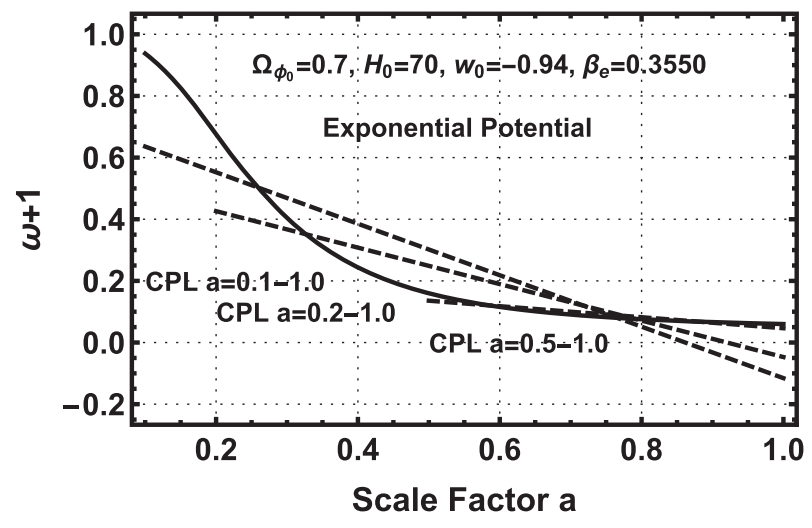

Figure 25. Three CPL fits, dashed lines, to the evolution of $w(a)$ for an exponential potential with $w_{0}=-0.94$. The fits to the full range and the range between $a=0.2$ and 1.0 produce false phantom crossings.

suggest a possible reason why many observational studies seem to favour phantom, $w<-1$, values of $w$ e.g. (Chen, Huang \& Wang 2017). Fig. 25 shows CPL fits to the $w_{0}=-0.94 w(a)$ evolution for an exponential potential over three scale factor ranges; the full range between 0.1 and $1.0(z=9-0)$, the range between 0.2 and $1.0(z=4-0)$, and the range between 0.5 and $1.0(z=1-0)$.

As expected the fit between $z=1$ and 0 is a good match but the two fits that include the higher redshift evolution produce phantom values for $w_{0}$ in equation (45) even though the true evolution has no phantom values. It is also evident that observations at redshifts greater than one provide more leverage on constraining deviations of $w(a)$ from minus one than observations between redshifts one and zero.

Measurements of the values of $\mu$ and $\alpha$ provide more precise constraints on dynamical dark energy. Figs 17 and 18 show the expected evolutionary tracks for $\mu$ with a coupling constant $\zeta_{\mu}=$ $\pm 10^{-6}$ and the five different values of $w_{0}$. A single measurement, under the quintessence assumption of homogeneous dark energy, determines the allowed parameter space for dynamical dark energy. Figs 19 and 20 show the allowed parameter space in the $\left(w_{0}+1\right)$, $\zeta_{\mu}$ plane based on the observational constraint discussed in Section 9.1.1. Any point other than 0,0 in the plane requires dynamical dark energy, new physics or both.

\subsection{The dark energy potential}

As with the question of dynamical versus static dark energy, the Hubble parameter yields essentially no information on the functional form of the dark energy potential. Although not explicitly depicted here the tracks of the cosmological parameters, such as $w(a)$ for the logarithmic potential have the same insensitivity to the value of $\beta_{l}$ as shown for the power and inverse power-law potential in paper I. The $w(a)$ tracks for the exponential potential, however, are sensitive to $\beta_{\mathrm{e}}$ since the values of $\beta_{\mathrm{e}}$ and $w_{0}$ are coupled by equation (16).

An accurate observational measurement of $w(a)$ at a particular scale factor or for a range of scale factors does not uniquely determine the dark energy potential. Examination of Figs 15 and 16 shows that for a given coordinate in the $w(a), a$ plane either a logarithmic or exponential potential can match the coordinate by altering the value of $w_{0}$. The tracks in the two figures are for specific values of $w_{0}$ but all of the area between the minimal and maximal tracks are covered by the range of $w_{0}$ between -0.9 and -0.98 . All of the area below the minimal -0.98 can be covered by making

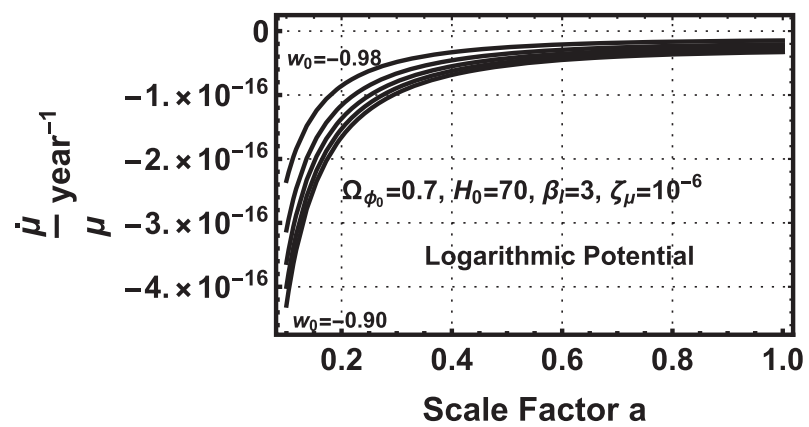

Figure 26. The rate of change, $\dot{\mu} / \mu$ per year for the logarithmic potential with a coupling constant of $\zeta_{\mu}=10^{-6}$.

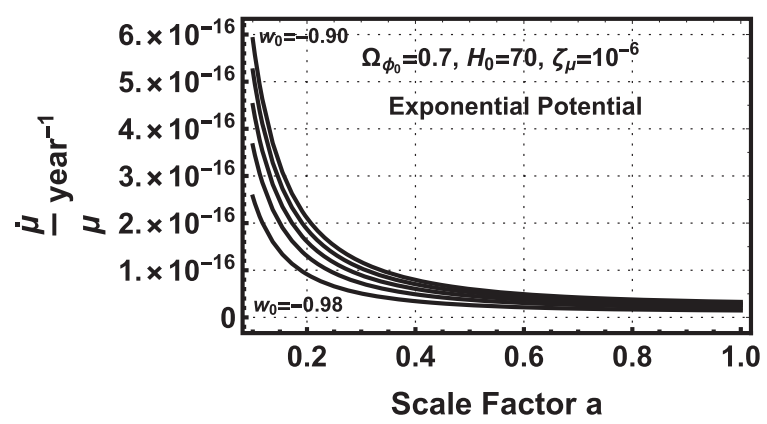

Figure 27. The rate of change, $\dot{\mu} / \mu$ per year for the exponential potential with a coupling constant of $\zeta_{\mu}=10^{-6}$.

$w_{0}$ arbitrarily close to -1.0 and the area above the maximal -0.9 tracks can be covered by making $w_{0}$ even further from -1 . The tracks in both figures have very similar shapes, making it difficult to discriminate between the potentials even with good knowledge of $w(a)$ over a large range of scale factors. However, if there is an accurate measurement of $w_{0}$ along with $w(a)$ at other scale factors there is some leverage in determining the potential. Of course any determination of $w$ other than minus one at any epoch would be a significant finding.

\subsection{The rate of change of fundamental constants}

Laboratory constraints on the rate of change of fundamental constants is another check on the possibility of dynamical dark energy. Figs 17 and 18 indicate that the rate of change of $\mu$ and $\alpha$ in a quintessence freezing cosmology is slowing down in the current epoch. Figs 26 and 27 show the rate of change, $\dot{\mu} / \mu$ per year for the logarithmic and exponential dark energy potentials with a coupling constant of $\zeta_{\mu}=10^{-6}$. The proton-to-electron ratio is used in the example but the fine structure constant $\alpha$ has exactly the same track if its coupling constant is also $\zeta_{\alpha}=10^{-6}$. It is clear from Figs 26 and 27 that in a quintessence freezing cosmology the current rate of change of the fundamental constants is significantly less than rate at high redshift. Table 1 shows the rate of change in units of $10^{-17} \mathrm{~m}_{\mathrm{pl}}$ per year for $\mu$ at scale factors of 0.1 and 1.0 for the logarithmic and exponential potentials for the five values of $w_{0}$ and a coupling constant of $+10^{-6}$. The signs between the two potentials are opposite and would be reversed for a negative coupling constant. The current rates of change are essentially the same between the two potentials but diverge at a scale factor of 
Table 1. $\dot{\mu} / \mu$ per year for the logarithmic and exponential dark energy potentials at scale factors of 0.1 and 1.0 for the five values of the current dark energy equation of state $w_{0}$ and a coupling constant of $\pm 10^{-6}$.

\begin{tabular}{lcccc}
\hline & \multicolumn{4}{c}{$\dot{\mu} / \mu$ in $10^{-17} m_{\mathrm{pl}}$ per year } \\
& \multicolumn{2}{c}{ Logarithmic } & \multicolumn{2}{c}{ Exponential } \\
$w_{0}$ & $\mathrm{a}=0.1$ & $\mathrm{a}=1.0$ & $\mathrm{a}=0.1$ & $\mathrm{a}=1.0$ \\
\hline-0.98 & -23.4 & -1.47 & 25.6 & 1.47 \\
-0.96 & -31.1 & -2.07 & 36.5 & 2.07 \\
-0.94 & -36.2 & -2.54 & 45.0 & 2.54 \\
-0.92 & -40.0 & -2.93 & 52.4 & 2.93 \\
-0.90 & -42.9 & -3.28 & 59.0 & 3.28 \\
\hline
\end{tabular}

Table 2. The two swampland parameters for the potentials in this paper and paper I. The units of $\Delta \phi$ are Planck masses. The $\beta_{l, p, i}$ values are 3 for all potentials except for the exponential potential which uses the $\beta_{\mathrm{e}}$ value appropriate to the $w_{0}$ value.

\begin{tabular}{lccccccccc}
\hline \multicolumn{1}{c}{ Log } & \multicolumn{1}{c}{ Swampland parameters } \\
\multicolumn{1}{c}{$w_{0}$} & $\Delta \phi$ & $\frac{\Delta V}{V}$ & $\Delta \phi$ & $\frac{\Delta V}{V}$ & $\Delta \phi$ & $\frac{\Delta V}{V}$ & $\Delta \phi$ & $\frac{\Delta V}{V}$ \\
\hline-0.98 & 0.45 & 0.09 & -0.47 & 0.10 & 0.55 & 0.10 & -0.58 & 0.11 \\
-0.96 & 0.62 & 0.18 & -0.67 & 0.21 & 0.76 & 0.20 & -0.84 & 0.23 \\
-0.94 & 0.73 & 0.26 & -0.82 & 0.34 & 0.92 & 0.30 & -1.06 & 0.38 \\
-0.92 & 0.82 & 0.34 & -0.94 & 0.47 & 1.04 & 0.41 & -1.26 & 0.56 \\
-0.90 & 0.90 & 0.42 & -1.06 & 0.62 & 1.14 & 0.52 & $\mathrm{X}$ & $\mathrm{X}$ \\
\hline
\end{tabular}

0.1 . The average current rate of change is roughly 18 times less than the rate of change at a scale factor of 0.1. Current laboratory bounds (Godun et al. 2014) are $\dot{\mu} / \mu=(0.2 \pm 1.1) 10^{-16} \mathrm{yr}^{-1}$ and $\dot{\alpha} / \alpha=(-0.7 \pm 2.1) 10^{-17} \mathrm{yr}^{-1}$. Matching the cosmological observational bounds on $\Delta \mu / \mu$ discussed in Section 9.1.1 with a coupling constant of $\pm 10^{-6}$ requires $\left(w_{0}+1\right) \leq 0.02$ which is the first row in Table 1. This sets a limit a factor of 10 below the laboratory limit. Unlike the laboratory limits the cosmological limit on $\Delta \mu / \mu$ is more stringent than the limit on $\Delta \alpha / \alpha$.

\subsection{Checking on the swampland}

String theory postulates a vast landscape of vacua that is surrounded by an even more vast landscape, termed the swampland, of consistent looking scalar field theories that are inconsistent with a quantum field theory of gravity (Vafa 2005; Agrawal et al. 2018). Put another way the swampland is the landscape of valid scalar field theories that are incompatible with quantum gravity (Heisenberg et al. 2018). Given the current interest in the swampland it is worthwhile to determine whether quintessence with the potentials considered here and in paper I dwells in the swampland. The boundaries of the swampland are usually defined by two conjectures. The first conjecture is that the change in the scalar should be $\Delta \phi<\sim O(1)$ and the second is that $\Delta V / V \geq \sim O(1)$. If either of these conjectures are violated then the cosmology is in the swampland. It is not entirely clear how restrictive of order 1 is or exactly what range of scale factors $\Delta \phi$ and $\Delta V$ encompass. It is obvious that $\Lambda C D M$ is in the swampland since $\Delta V=0$.

The quintessence models considered here and in paper I certainly live near the swampland with perhaps one foot in the swamp and one foot dry depending on how of order 1 is interpreted. The swampland parameters for the potentials in this paper and paper I are shown in Table 2 . Both $\Delta$ values are for the scale factor range between 0.1 and 1.0. The potential $V$ in $\Delta V / V$ is the current-day potential. The $\mathrm{X}$ in the inverse power-law parameters for $w_{0}=-0.90$ indicate that this is not a valid solution as shown in paper I.

All of the exponential and logarithmic potential cases considered here satisfy the condition on $\Delta \phi$ under the assumption that -1.06 is of order 1 . The power and inverse power law $\Delta \phi$ entries for the three values of $w_{0}$ closest to minus one, the most likely values, also satisfy the $\Delta \phi$ conjecture, the dry foot. None of the $\Delta V / V$ entries strictly satisfy the associated conjecture, the wet foot. Very recent work by Kinney, Vagnozzi \& Visinelli (2018) suggest that this is a feature common to most single scalar field cosmologies. Since the potentials $V(\phi)$ are functions of the scalar $\phi$ larger values of $\Delta V$ require larger changes in $\phi$ which, as Table 2 shows, requires larger deviations of $w$ from minus one and drives the $\Delta \phi$ values higher which could result in violating the $\Delta \phi$ conjecture. Obied et al. (2018) have also suggested a criterion that $|\phi|<1$ in Planck units which is not satisfied by the scalars in this work. It is not the purpose of this discussion to determine whether having one foot in the swamp is a good or bad thing but rather to simply show where quintessence with the potentials examined here lies with respect to the swampland boundaries.

\section{CONCLUSIONS}

This and paper I show that the beta function formalism provides an effective way to calculate accurate solutions for cosmological parameters as a function of the scale factor $a$. For the most part the solutions are analytic functions utilizing known mathematical functions. The superpotential for the logarithmic dark energy potential, however, required an easily calculated numerical integral. The two papers also demonstrate the application of the beta function formalism and can act as a guide to the extension of the formalism to other potentials and cosmologies.

\section{REFERENCES}

Agrawal P., Obied G., Stenhardt P. J., Vafa C., 2018, Phys. Lett, 784, 27 Avsajanishvilli O., Huang Y., Samushia L., Kahniashvilli T., 2017, Eur. Phys. J., 78, 773

Bagdonaite J., Dapra, Jansen P., Bethlem H. L., Ubachs W., Henkel C., Menten K. M., 2013, Phys. Rev. Lett., 111, 231101

Binetruy P., Kiritisis E., Mabillard J., Pieroni M., Rosset C., 2015, JCAP, 1504, 033

Chen L., Huang Q.-G., Wang K., 2017, Eur. Phys. J., 77, 762

Chevallier M., Polarski D., 2001, Int. J. Mod. Phys., 10, 213

Cicciarella F., Mabillard J., Pieroni M., 2018, JCAP, 1, 24

Cicciarella F., Pieroni M., 2017, JCAP, 1708, 10

Godun R. M. et al., 2014, Phys. Rev. Lett., 113, 210801

Heisenberg L., Bartelmann M., Brandenberger R., Refreiger A., 2018, preprint ( astro-ph/1808.02877v2)

Kanekar N. et al., 2015, MNRAS, 448, L104

Kinney W. H., Vagnozzi S., Visinelli L., 2018, preprint ( astro$\mathrm{ph} / 1808.06424 \mathrm{v} 1)$

Linder E. V., 2003, Phys. Rev. Lett., 90, 091301

Narain G., 2018, International Journal of Modern Physics A, 33, 1850031

Nunes N. J., Lidsey J. E., 2004, Phys. Rev., 69, 123511-1-7

Obied G., Ooguri H., Spodyneiko L., Vafa C., 2018, preprint (arXiv:1806.0 $8362 \mathrm{v} 2)$

Scherrer R. J., Sen A. A., 2008, Phys. Rev., 083515

Thompson R. I., 2018, MNRAS, 477, 4104

Vafa C., 2005, preprint (arXiv:hep-th/0509212v2)

This paper has been typeset from a $\mathrm{T}_{\mathrm{E}} \mathrm{X} / \mathrm{L} \mathrm{A} \mathrm{T}_{\mathrm{E}} \mathrm{X}$ file prepared by the author. 